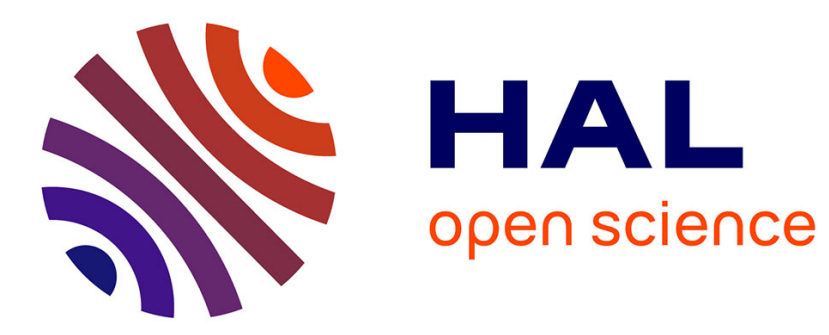

\title{
Dynamic Behavior Investigation for Trajectory Control of a Microrobot in Blood Vessels
}

Laurent Arcèse, Ali Cherry, Matthieu Fruchard, Antoine Ferreira

\section{To cite this version:}

Laurent Arcèse, Ali Cherry, Matthieu Fruchard, Antoine Ferreira. Dynamic Behavior Investigation for Trajectory Control of a Microrobot in Blood Vessels. 2010 IEEE International Conference on Intelligent Robots and Systems, Oct 2010, Taipei, Taiwan. pp 5774-5779. hal-00655998

\section{HAL Id: hal-00655998 \\ https://hal.science/hal-00655998}

Submitted on 3 Jan 2012

HAL is a multi-disciplinary open access archive for the deposit and dissemination of scientific research documents, whether they are published or not. The documents may come from teaching and research institutions in France or abroad, or from public or private research centers.
L'archive ouverte pluridisciplinaire HAL, est destinée au dépôt et à la diffusion de documents scientifiques de niveau recherche, publiés ou non, émanant des établissements d'enseignement et de recherche français ou étrangers, des laboratoires publics ou privés. 


\title{
Dynamic behavior investigation for trajectory control of a microrobot in blood vessels
}

\author{
Laurent Arcese, Ali Cherry, Matthieu Fruchard, Antoine Ferreira
}

\begin{abstract}
This paper reports modeling and control of a microsized polymer aggregate of magnetic particles inside an artery, using a MRI device for supplying propulsion in order to achieve targeted chemotherapy. Non-newtonian behaviour of blood is took into account, as well as wall effects and interactions, resulting in a highly nonlinear model. A backstepping approach is synthesized to ensure Lyapunov stability along a pre-planned trajectory inherited from the model, with robustness concerns.
\end{abstract}

\section{INTRODUCTION}

Autonomous microrobots designed to perform targeted therapy by navigating in the cardiovascular system are a prolific research area for minimally invasive surgery purposes [1]. Untethered robots are particularly well suited either for reaching remote parts of the body which remain inaccessible to present tools without operating, or for targeting therapy in such places. In the case of chemotherapy, such robots carrying drugs can both improve the therapy's efficiency, reduce drug dose and in turn side effects. On such a scale, it proves difficult to embed actuators sufficiently powerful to propel the robot, especially when swimming against the tide. Therefore, the great majority of swimming approaches considers magnetic fields to wirelessly transmit power to the robot [4], [7].

Bead pulling has first been studied using magnets [15] or superconducting magnets [16]. The last innovation in this domain has been provided by Martel [12], where the basic idea is to use the gradients coils of a clinical MRI to pull the robot. Since medical applications are the underlying theme of these works, using a MRI device to actuate the robot combines several advantages. MRI devices are widely implanted and provide magnetic gradients that can be used for propelling purposes. Besides, the MRI imager can provide fine observation of the scene for navigation, using multiplexing for controlling and imaging [17].

What is the optimal size for such a robot? On the one hand -from a therapeutic viewpoint- the smaller the better, so as to avoid embolization far from the tumor and to drive the drugs as close as possible to the tumor. On the other hand, since magnetic forces are volumic, they decrease in

L. Arcese and M. Fruchard are with the Institute PRISME UPRES 4229, IUT de Bourges, 63 Av de Lattre de Tassigny, 18020, Bourges cedex, France laurent.arcesedbourges.univ-orleans.fr matthieu.fruchardebourges. univ-orleans.fr

A. Cherry and A. Ferreira are with the Institute PRISME UPRES 4229, ENSI de Bourges, 88 Bd Lahitolle, 18000, Bourges, France ali.cherrydensi-bourges.fr antoine.ferreiradensi-bourges.fr cubic with size. Besides, partial vessel occlusion by the robot results in an optimal ratio between robot's and vessel's radii evocated in [12]. Along the reference trajectory, from injection point to tumor's area, vessels radius decreases. It is thus necessary to make the radius of the robot decrease as it goes in smaller vasculature. This can be done if the robot is a polymer binded aggregate of magnetic particles, loosing substance with time.

This paper deals with both modeling and controlling such a robot along a reference trajectory. This contribution mainly relies on defining a precise forces balance (Section II), which includes wall effects (parabolic profile of blood flow, pulsatile arterial walls and effect of the ratio of robot's on vessel's radii), wall interactions (Van der Waals, contact and electrostatic forces) and non-newtonian behaviour of blood. The goal is not only to describe the robot's behaviour accurately, but also to make a link with off-line approaches [20] without their time-consuming drawbacks. An optimal reference trajectory is hence deduced from the nonlinear model (Section III), in order to minimize the control efforts.

A state space representation is then inherited from the forces balance model (Section IV), so as to synthesize a backstepping control law (Section V). To our knowledge, most of feedback controller schemes designed in the scope of microrobotics facing drag are PID approaches [17], [13]. Authors report instabilities and important oscillations around the equilibrium, especially when the blood stream is modeled as a pulsatile flow. They also indicate a lack of robustness to noise and unmodeled dynamics. In this paper, the control design is based on the backstepping approach recently developed in [3], which ensures Lyapunov stability. Since backstepping can be regarded as a pre-compensation of the model's nonlinearities, working with a more accurate model is a real improvement of our previous work. Some simulations results illustrate the strengths and limitations of the overall study (Section VI). Lastly we sum up results and discuss prospects (Section VII).

\section{Modeling: Forces BALANCE}

The purpose of this section is to present a $2 \mathrm{D}$ state space model of a ferromagnetic microrobot of radius $r$ immersed in blood vessel. The model encompasses the different forces that affect the robot's motion as well as its interaction with the vessel wall. The translational and rotational motions of the robot are expressed by:

$$
\left\{\begin{array}{l}
m \frac{d \vec{v}}{d t}=\vec{F}_{m}+\vec{F}_{d}+\vec{W}_{a}+\vec{F}_{c}+\vec{F}_{v d w}+\vec{F}_{\text {elec }} \\
J \frac{d \vec{w}}{d t}=\vec{T}_{m}+\vec{T}_{d}+\vec{M}_{c}
\end{array}\right.
$$


where $\vec{v}$ and $\vec{w}$ are, respectively, the translational and rotational velocity of the robot, $m$ and $J$ its mass and moment of inertia. $\vec{F}_{m}, \vec{F}_{d}, \vec{W}_{a}, \vec{F}_{c}, \vec{F}_{v d w}$ and $\vec{F}_{\text {elec }}$ respectively denote the magnetic force produced by the MRI gradient coils, blood hydrodynamic drag force, apparent weight, the robot-to-wall contact force, the Van der Waals force and the electrostatic force. $\vec{T}_{m}, \vec{T}_{d}$ and $\vec{M}_{c}$ denote respectively the magnetic torque, hydrodynamic drag torque and the robotto-wall contact moment.

In the rest of this paper, we assume that the orientation of the robot does not change due to the magnetic torque which tends to align the magnetization of the robot along the external field. $\vec{T}_{d}$ and $\vec{M}_{c}$ are much smaller order than $\vec{T}_{m}$.

To determine whether statistical mechanics or continuum mechanics formulations of fluid dynamics should be used, we refer to the dimensionless Knudsen number $K_{n}=\frac{k_{b} T}{P \pi r^{3} 2^{5 / 2}}$, where $T, k_{b}$ and $P$ respectively denote temperature, the Boltzmann constant and pressure. In our case, the low value $K_{n} \approx 10^{-13}\left(\ll 10^{-3}\right)$ ensures that the robot is large enough to neglect the effect of Brownian motion: the robot's motion is subjected to generic Navier-Stokes equations.

\section{A. Magnetic force}

The gradient coils of the MRI system provide magnetic gradients which produce a magnetic force $F_{m}$ on the robot:

$$
\vec{F}_{m}=\tau_{m} \mu_{0} V(\vec{M} \cdot \nabla) \vec{H}
$$

where $V$ is the robot's total volume, $\tau_{m}=\frac{V_{m}}{V}$ with $V_{m}$ the ferromagnetic volume, $\vec{M}$ is the magnetization of the material, $\mu_{0}$ is the permeability of free space, $\vec{H}$ is the external magnetic field and $\nabla$ is the gradient operator.

\section{B. Hydrodynamic drag force}

The hydrodynamic drag force $\vec{F}_{d}$ exerting on a spherical body in an infinite extent of fluid is expressed as:

$$
\vec{F}_{d}=-\frac{1}{2} \rho_{f}\left(\vec{v}-\vec{v}_{f}\right)_{\infty}^{2} A C_{d_{\infty}}
$$

where $\vec{v}-\vec{v}_{f}$ denotes the relative velocity of the robot with respect to the fluid, $A$ is the frontal area of the core and $\rho_{f}$ is the density of the fluid. $C_{d_{\infty}}$ is the drag coefficient, a dimensionless quantity used to quantify the drag or resistance of an object in a fluid. $C_{d_{\infty}}$ is a function of the Reynolds number $R e_{\infty}=\frac{2 r \rho_{f}\left|\vec{v}-\vec{v}_{f}\right|_{\infty}}{\eta}$; its expression for a spherical body in laminar flow is given by [21]:

$$
C_{d_{\infty}}=\frac{24}{R e_{\infty}}+\frac{6}{1+\sqrt{R e_{\infty}}}+0.4
$$

1) Non-Newtonian fluid: In case of blood, which exhibits a non-newtonian behavior, the fluid's viscosity $\eta$ is a function of vessel diameter $d$ (in micron) and hematocrit rate $h_{d}$ according to the following empirical relations [14]:

$$
\eta=\frac{\eta_{\text {plasma }} d^{2}}{(d-1.1)^{2}}\left[1+\frac{\left(\eta_{0.45}-1\right) d^{2}}{(d-1.1)^{2}} \frac{\left(1-h_{d}\right)^{c}-1}{(1-0.45)^{c}-1}\right]
$$

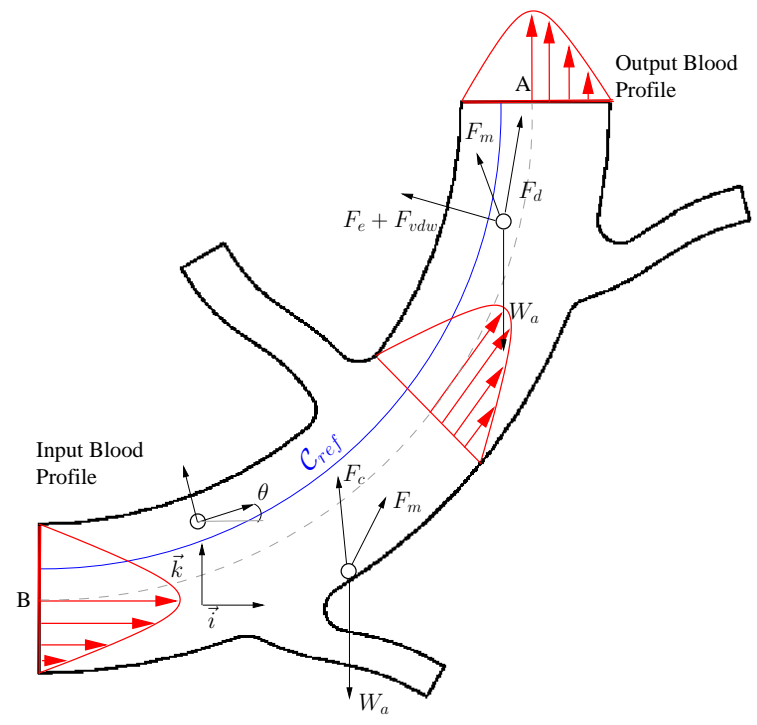

Fig. 1. Scheme of a blood vessel with minor bifurcations

with parameters $\eta_{\text {plasma }}$ and $\eta_{0.45}$ denoting respectively the plasma's viscosity and the relative apparent blood viscosity for a fixed discharge hematocrit of 0.45 , given by:

$$
\eta_{0.45}=6 e^{-0.085 d}+3.2-2.44 e^{-0.06 d^{0.645}}
$$

The shape of the viscosity dependance on hematocrit is:

$$
c=\frac{10^{11}}{d^{12}}-\left(0.8+e^{-0.075 d}\right)\left(\frac{d^{12}}{d^{12}+10^{11}}\right)
$$

2) Wall effects: For endovascular applications, influence of the vessel walls on the velocity of the robot has to be taken into account. In general, this wall effect is expressed as a ratio between the terminal relative velocity of the robot $\left(\vec{v}-\vec{v}_{f}\right)_{t}$ and its velocity $\left(\vec{v}-\vec{v}_{f}\right)_{\infty}$ in an infinite extent of fluid [10]:

$$
\frac{\left|\vec{v}-\vec{v}_{f}\right|_{t}}{\left|\vec{v}-\vec{v}_{f}\right|_{\infty}}=\frac{1-\lambda^{\alpha_{0}}}{1+\left(\frac{\lambda}{\lambda_{0}}\right)^{\alpha_{0}}}
$$

with ratio $\lambda=2 r / D$ and $D$ denoting the vessel diameter (in meter). Parameters $\alpha_{0}$ and $\lambda_{0}$ are functions of Reynolds number, but are commonly set to 1.5 and 0.29 , respectively.

Thus, equation (3) is corrected as follows:

$$
\vec{F}_{d}=-\frac{1}{2} \rho_{f}\left[\left(\frac{1+\left(\frac{\lambda}{\lambda_{0}}\right)^{\alpha_{0}}}{1-\lambda^{\alpha_{0}}}\right)\left(\vec{v}-\vec{v}_{f}\right)_{t}\right]^{2} A C_{d_{t}}
$$

Wall effects on the fluid in the vessel traditionally result in a parabolic profile of blood flow (see Figure 1). Besides, to fully take into account pulsatile flow caused by heart pumping in arteries, one has to consider a periodic deformation of the vessel's diameter $D(t)$ synchronized with $v_{f}(t)$. 

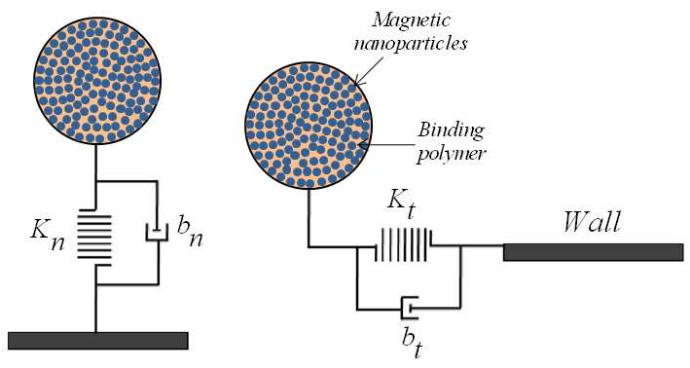

Fig. 2. Model of contact forces: robot-to-wall

\section{Apparent weight}

In addition to the magnetic and hydrodynamic forces, apparent weight (combined action of weight and buoyancy) is acting on the spherical robot:

$$
\vec{W}_{a}=V\left(\rho-\rho_{f}\right) \vec{g}
$$

where $\rho=\tau_{m} \rho_{m}+\left(1-\tau_{m}\right) \rho_{\text {poly }}$ with $\rho_{m}$ and $\rho_{\text {poly }}$ the magnetic material's and polymer's densities.

\section{Contact force}

The normal and tangential interaction between the robot and the wall are assumed to be expressed by a Voigt model with the spring constant $K$ and the decay coefficient of dashpot $b$, as shown in Figure 2, where the indexes $\vec{n}$ and $\vec{t}$ indicate respectively the normal and tangential normalized vectors.

The normal component of the contact force $\vec{F}_{c n}$ acting on the robot is given by the sum of a non-linear elastic component and a linear viscous damping: [18]:

$$
\vec{F}_{c n}=\left(K_{n} \delta^{3 / 2}+b_{n} \dot{\delta}\right) \vec{n}
$$

where $\delta$ is the elastic deformation of the wall at the contact point and $\vec{n}$ is the normal unit vector pointing from the robot to the contact surface. The stiffness $K_{n}$ can be calculated by Hertzian contact theory [19] as follows:

$$
K_{n}=\frac{\frac{4 \sqrt{r}}{3}}{\frac{1-\sigma_{p}^{2}}{E_{p}}+\frac{1-\sigma_{w}^{2}}{E_{w}}}
$$

where $E_{p}$ and $E_{w}$ are the Young's modulus of robot and wall, and $\sigma_{p}, \sigma_{w}$ are respectively the Poisson's ratios of robot and wall. The damping coefficient $b_{n}$ is deduced from $K_{n}$ using $b_{n}=2 \sqrt{m K_{n}}$.

The tangential component of the contact force takes part when the robot is rotating or in case of oblique collision with the wall:

$$
\vec{F}_{c t}=\left(K_{t} \zeta+b_{t} \dot{\zeta}\right) \vec{t}
$$

where $\zeta$ is the displacement in the tangential direction and $\vec{t}$ is the tangential unit vector. The stiffness $K_{t}$ and damping coefficient $b_{t}$ are given by:

$$
K_{t}=\frac{8 \sqrt{r} G_{p}}{2-\sigma_{p}} \delta^{1 / 2}, \quad b_{t}=2 \sqrt{m K_{t}}
$$

where $G_{p}$ is the shear modulus related to the Young's modulus $E_{p}$ and Poisson ratio $\sigma_{p}$, i.e. $G_{p}=\frac{E_{p}}{2\left(1+\sigma_{p}\right)}$.

\section{E. Van der Waals and electrostatic forces}

When the robot and the wall are not in contact, they interact each other through Van der Waals and electrostatic forces. These two interaction forces have different dominant regimes. In fact, when the robot is close to the wall, Van der Waals force is dominant. As the robot move away from the wall, the Van der Waals force rapidly decrease and the electrostatic force becomes dominant.

The Van der Waals potential between the robot and the wall is given by [8]:

$$
\vec{V}_{v d w}=-\frac{A_{h}}{6}\left(\frac{1}{h}+\frac{1}{2+h}+\ln \frac{h}{2+h}\right) \vec{n}
$$

where $A_{h}$ is the Hamaker constant and $h$ is the distance between robot and wall. Then, the Van der Waals interaction force is given by differenciating (9) $\vec{F}_{v d w}=-\nabla \vec{V}_{v d w}$. The electrostatic force between the robot and the wall considered as an uncharged surface is given by [5]:

$$
\vec{F}_{\text {elec }}=\frac{q^{2}}{4 \pi \epsilon \epsilon_{0}(r+h)^{2}} \vec{n}
$$

with $q$ the robot charge, $\epsilon$ the dielectric density of the medium in which the interaction occurs and $\epsilon_{0}$ the vacuum permittivity.

[6] gives the expression of the maximum allowable charge $Q=30 \times(100 r)^{-0.3}$ for a spherical body of radius $r$.

\section{Modeling: Optimal Trajectory}

Previous forces balance gives us sufficient informations to plan an optimal trajectory. At least two cases A and B, shown on the Figure 1, should be taken into account. In the first one, the robot is in a vertical vessel and the magnetic force $\vec{F}_{m}$ should counter both contributions of the robot's apparent weight $\vec{W}_{a}$ and the drag force $\vec{F}_{d}$ when blood is flowing back (Curve A of Figure 3). The drag force decreases when the robot approaches the wall due to the parabolic profile of velocity. Thus the reference trajectory should be as near as possible to wall. In the second case, the robot is in a horizontal vessel and the magnetic force should counter contributions of the robot's apparent weight, electrostatic and Van der Waals forces, $\vec{F}_{\text {elec }}$ and $\vec{F}_{v d w}$ (Curve B of Figure 3). This case shows that near the wall, $\vec{F}_{\text {elec }}$ and $\vec{F}_{v d w}$, which point to the wall, are dominant and the magnetic force is no more sufficient to counter it. Nevertheless there is an optimal position where the sum of the two forces compensates perfectly the robot's weight. Moreover, the curve A shows that at this point, the magnetic force exceeds the drag force. From these observations, we define an optimal path as an arc passing through the point $\mathrm{C}$.

\section{Modeling: State Space Representation}

Let $(x, z)$ denote the position of the robot in the vessel with respect to a given frame $\mathcal{F}(O, \vec{i}, \vec{k})$. The state model is established from differential equation (1) defining the robot's dynamic behavior, projected on $\vec{i}$ and $\vec{k}$ axes:

$$
\left\{\begin{array}{l}
m \ddot{x}=F_{m_{x}}+F_{d_{x}}+F_{c n_{x}}+F_{c t_{x}}+F_{v d w_{x}} \\
m \ddot{z}=F_{m_{z}}+F_{d_{z}}+W_{a}+F_{c n_{z}}+F_{c t_{z}}+F_{v d w_{z}}
\end{array}\right.
$$




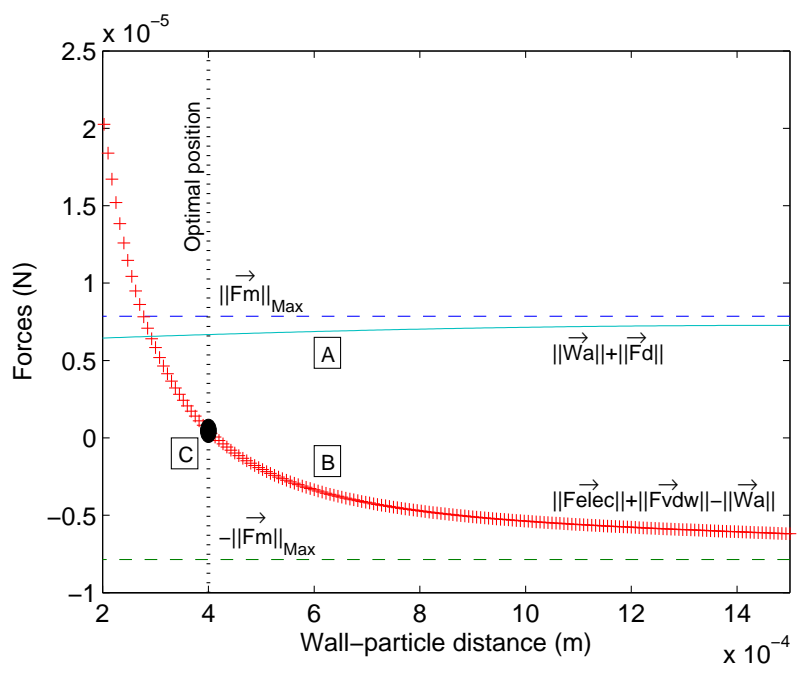

Fig. 3. Forces balances in vertical (A) and horizontal (B) artery

where indexes $x$ (respectively $z$ ) denote projections on $\vec{i}(\vec{k})$.

Let $x_{1}, x_{2}, x_{3}, x_{4}$ denote respectively the particule's position and velocity along $\vec{i}$ axis, and the position and velocity along $\vec{k}$ axis. Assuming that positions $x_{1}$ and $x_{3}$ can be measured thanks to the MRI imaging system, let $y$ denote the state's measure. Using expressions of forces given by (2), (5), (6), (7), (8), (10) and Van der Waals interaction derivated from potential (9), and adequate projection of local frame along the geometry of the vessel ${ }^{1}$, system (11) can be written in the form:

$$
(\mathcal{S})\left\{\begin{array}{l}
\dot{x}_{1}=x_{2} \\
\dot{x}_{2}=f_{2}\left(x_{1}, x_{2}, x_{3}, x_{4}\right)+a u_{1} \\
\dot{x}_{3}=x_{4} \\
\dot{x}_{4}=f_{4}\left(x_{1}, x_{2}, x_{3}, x_{4}\right)+a u_{2} \\
y=\left(\begin{array}{c}
x_{1} \\
x_{3}
\end{array}\right)
\end{array}\right.
$$

where control inputs $u_{1}=\nabla B_{x}$ and $u_{2}=\nabla B_{z}$ are the magnetic gradients, parameter $a=\frac{\tau_{m} M}{\rho}$, and $f_{i}$ are very highly nonlinear functions of the full state.

\section{CONTROL APPROACH}

In this section, we present a control design for Lyapunovstabilizing trajectories for system $(\mathcal{S})$. Since determination of Lyapunov functions is generically a challenging issue, it is preferable to use Lyapunov control functions in a backstepping control approach [11], [9]. Since this design requires a triangular form for the control system, we propose the following change of coordinates:

$$
\left\{X=\left(\begin{array}{l}
x_{1} \\
x_{3}
\end{array}\right) ; \quad Y=\left(\begin{array}{l}
x_{2} \\
x_{4}
\end{array}\right) ; \quad U=\left(\begin{array}{l}
u_{1} \\
u_{2}
\end{array}\right)\right.
$$

Thus, we obtain a new system in a triangular form:

$$
\left(\mathcal{S}^{\prime}\right)\left\{\begin{array}{l}
\dot{X}=Y \\
\dot{Y}=F(X, Y)+U \\
y=X
\end{array}\right.
$$

\footnotetext{
${ }^{1}$ The drag force is not linearly distributed between $\vec{i}$ and $\vec{k}$ axis.
}

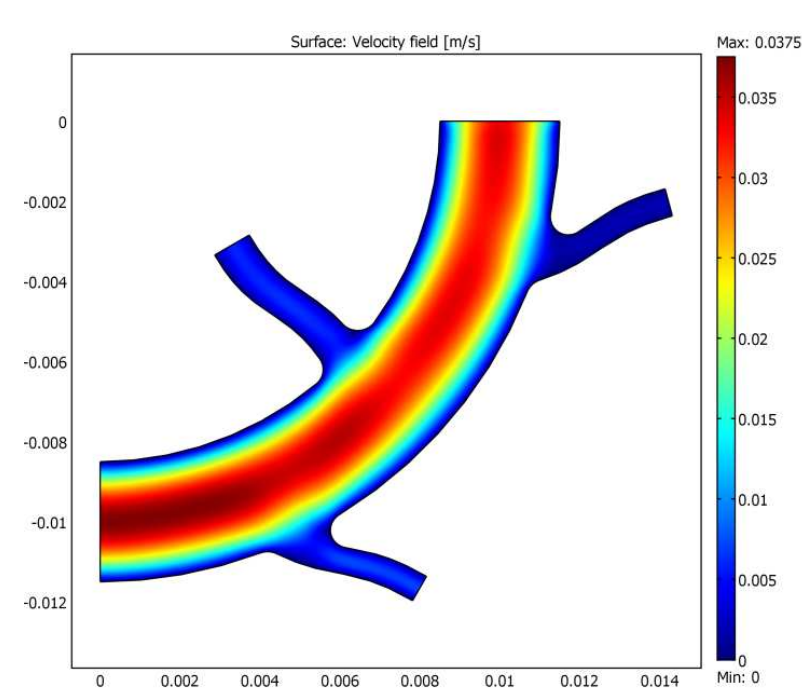

Fig. 4. Comsol results : Velocity profile in a blood vessel with minor bifurcations

Denoting $X_{\text {ref }}, \dot{X}_{\text {ref }}$ and $\ddot{X}_{\text {ref }}$ respectively the desired reference trajectory, velocity and acceleration, the control law for system $\left(\mathcal{S}^{\prime}\right)$ can be expressed as [3]:

$$
\begin{aligned}
U= & \ddot{X}_{r e f}+\left(k_{1}+k_{2}\right) \dot{X}_{r e f}+\left(1+k_{1} k_{2}\right) X_{r e f} \\
& -\left(1+k_{1} k_{2}\right) X-\left(k_{1}+k_{2}\right) Y-F(X, Y)
\end{aligned}
$$

where $k_{1}$ and $k_{2}$ are the strictly positive backstepping gains.

\section{SIMULATIONS}

The robot is made of $\tau_{m}=80 \% \mathrm{NdFeB}$ particles which has a combination of very high remanence and coercivity, and $20 \%$ of binding polymer. Simulations are performed by taking into account the limitations of a clinical MRI system. In order not to exceed the capacity of existing MRI systems, the applied control law (15) is now corrected as $\frac{u_{i}}{k(t)}$, with $k(t)=\max \left\{1, \frac{u_{i}}{u_{i, \max }}\right\}$.

A pulsatile flow is included by imposing a time-varying velocity. As a first approximation of a physiological pulse, we use a time-sinusoidal profile with spatial parabolic form. In the case of artery, such an approximation leads to:

$$
v_{f}(t)=0.035(1+1.15 \sin 2 \pi t) \times\left[1-\left(\frac{D / 2-h}{r}\right)^{2}\right]
$$

Our studies assume the presence of minor bifurcations (see Figure 1). This geometry leads to only slight change in the velocity's profile and amplitude (see Figure 4). The developed controller must be sufficiently robust to compensate this effect which could be considered as a disturbance. Major bifurcations will require a further study of velocity's field profile.

The purpose of the control is to ensure a null error between desired and a pre-planned trajectory. In the following, the performances and stability of the controller with respect to 

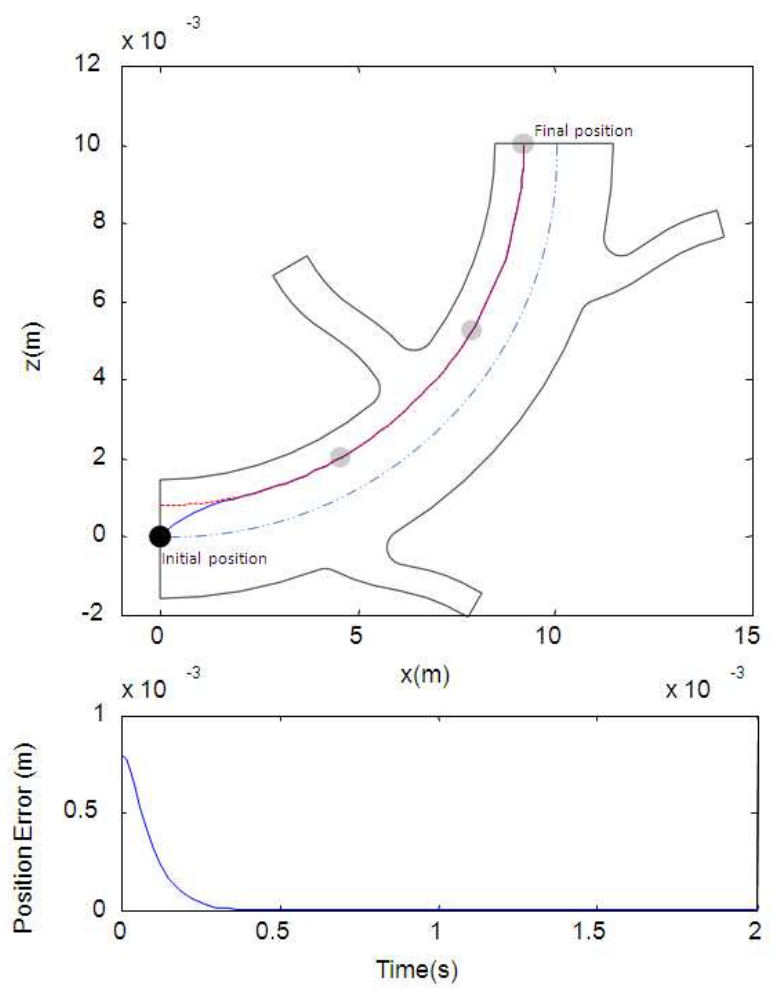

Fig. 5. XZ trajectory : reference trajectory (red dotted) and real trajectory (blue solid line)

noise measurement, parameters variations and uncertainties are illustrated by two simulations, whose parameters are given in Table I. A first simulation is led so as to pinpoint robustness to model errors, and the second one to study sensitivity to output noise.

Simulation of Figures 5, 6 is performed by assuming that the blood's viscosity and permittivity are affected by uncertainties of $100 \%$ of their nominal values and the vessel's diameter by uncertainties of $10 \%$. This simulation shows that after a transient phase, the position tracking performance is robust enough to model's error (subfigure of Figure 5). Despite these uncertainties, Figure 6 shows that the control inputs don't reach saturation. This is due to the choice of an optimal reference trajectory deduced from the analysis of Section III.

In a second simulation (Figures 7 and 8), we assume a white gaussian noise is applied on the position measurement. This noise is about $10 \%$ of the measured signal. Figure 7 illustrate that the controller is quite stable despite the noise, and does not even reach saturations (Figure 8). Nevertheless, it's clear that the system is sensitive to noise. We can expect that this drawback effect can be reduced using an observer. In addition to estimate some parameters like blood's velocity (assumed to be known in this paper), an observer will tend to smooth the output signals.

\section{CONCLUSION}

In this paper, we have presented a highly nonlinear model for a MRI guided microrobot in blood vessels. This model

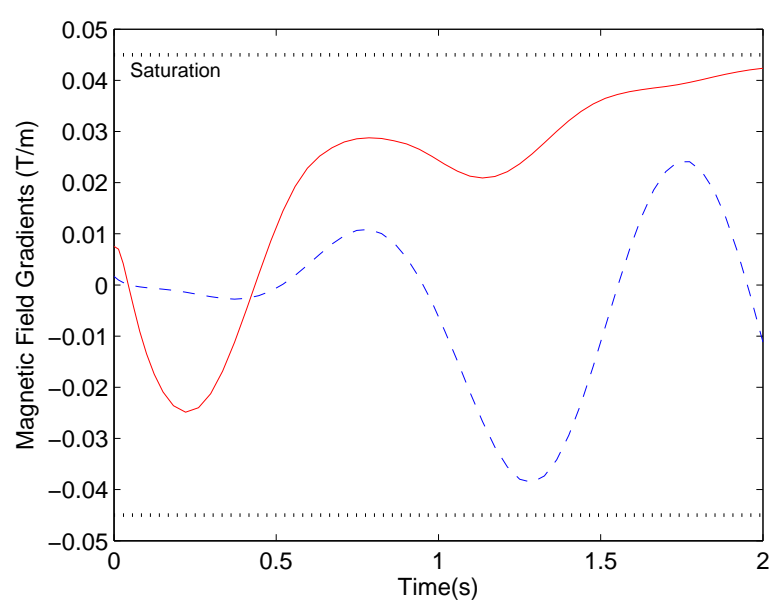

Fig. 6. Control input : magnetic field gradients on $\vec{i}$-axis (dotted) and on $\vec{k}$-axis (solid line)

TABLE I

SiMULATIONS DATA

\begin{tabular}{|c||c|c|}
\hline Plasma's viscosity & $\eta_{\text {plasma }}$ & $5 \times 10^{-3}[\mathrm{~Pa} . \mathrm{s}]$ \\
\hline Blood's density & $\rho_{f}$ & $1060\left[\mathrm{~kg} \cdot \mathrm{m}^{-3}\right]$ \\
\hline Robot's density & $\rho_{m}$ & $8000\left[\mathrm{~kg} \cdot \mathrm{m}^{-3}\right]$ \\
\hline Robot's radius & $r$ & $300[\mu \mathrm{m}]$ \\
\hline Vessel's diameter & $D$ & $3[\mathrm{~mm}]$ \\
\hline Polymer's density & $\rho_{\text {poly }}$ & $1500\left[\mathrm{~kg} \cdot \mathrm{m}^{-3}\right]$ \\
\hline Ferromagnetic ratio & $\tau_{m}$ & 0.8 \\
\hline Magnetization & $M$ & $1.95 \times 10^{6}\left[\mathrm{~A} \cdot \mathrm{m}^{-1}\right]$ \\
\hline Hematocrit & $h_{d}$ & 0.45 \\
\hline Robot's Young's modulus & $E_{p}$ & $10^{9}[\mathrm{~Pa}]$ \\
\hline Wall's Young's modulus & $E_{w}$ & $0.75 \times 10^{6}[\mathrm{~Pa}]$ \\
\hline Robot's Poisson's ratio & $\sigma_{p}$ & 0.27 \\
\hline Wall's Poisson's ratio & $\sigma_{w}$ & 0.30 \\
\hline Hamaker constant & $A_{h}$ & $4 \times 10^{-19}[\mathrm{~J}]$ \\
\hline Blood's dielectric density & $\varepsilon$ & $77\left[\mathrm{C}^{2} . \mathrm{N}^{-1} . \mathrm{m}^{-2}\right]$ \\
\hline Initial condition on $x$ & $x_{0}$ & $(0,0,0,0)^{T}$ \\
\hline Inputs saturations & $u_{i, m a x}$ & $45\left[\mathrm{mT}^{-1}\right]$ \\
\hline Controller gains & $k_{1}$ & 15 \\
\cline { 2 - 3 } & $k_{2}$ & 30 \\
\hline
\end{tabular}

takes into account the non-newtonian behavior of blood, as well as wall effects and interactions. It makes it possible to hence deduce an optimal trajectory. Besides, we have developed a nonlinear control law based on the backstepping approach. Parameters uncertainties and noise effects have been illustrated by simulations. It appears that the system is robust to uncertain physiological parameters, but proved quite sensitive to output noise, though remains stable.

As stated in Section VI, system robustness to noise can be improved by implementing a high gain observer based on [3] but fitted for this more complete and complex model. We are also working on estimating the blood's velocity and frequency, assumed to be known at the moment, using Kazantis-Kravaris Luenberger observers [2]. Additionnaly, the modeling of impact of major bifurcations on the blood's velocity profile is underway. 


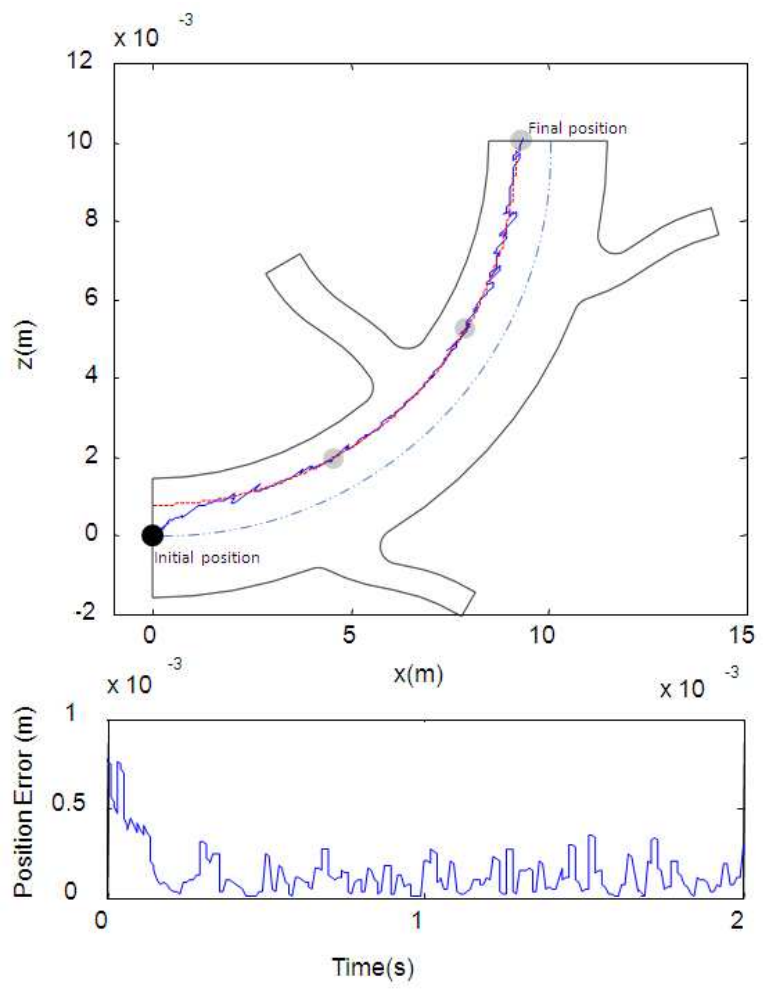

Fig. 7. XZ trajectory : reference trajectory (red dotted) and real trajectory (blue solid line)

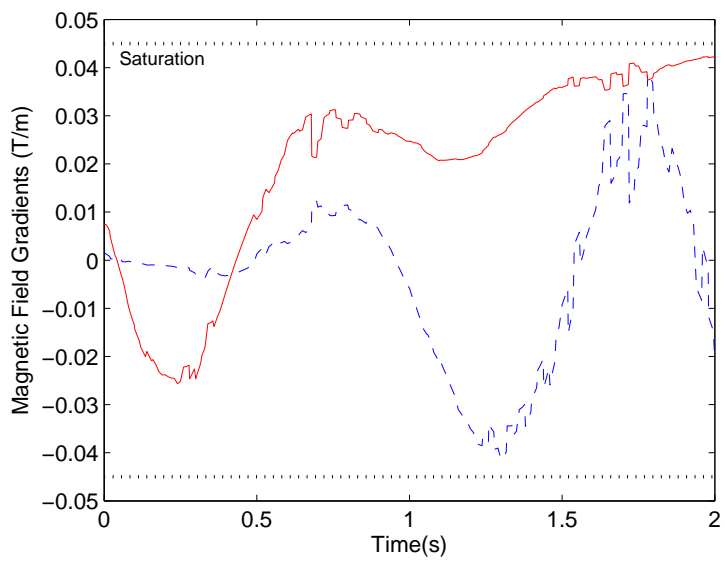

Fig. 8. Control input : magnetic field gradients on $\vec{i}$-axis (dotted) and on $\vec{k}$-axis (solid line)

\section{ACKNOWLEDGMENTS}

This work was supported by European Union's 7th Framework Program and its research area ICT-2007.3.6 Micro/nanosystems under the project NANOMA (NanoActuactors and Nano-Sensors for Medical Applications). The authors thank Christophe Boulnois for Comsol helpful advice.

\section{REFERENCES}

[1] J. Abbott, Z. Nagy, F. Beyeler, and B. Nelson, "Robotics in the small: Part 1 microrobotics," IEEE Robotics and Automation Magazine, vol. 14, no. 2, pp. 92-103, June 2007.
[2] V. Andrieu and L. Praly, "On the existence of a kazantis-kravaris / luenberger observer," SIAM, vol. 45, no. 2, pp. 432-456, 2006.

[3] L. Arcese, M. Fruchard, and A. Ferreira, "Nonlinear modeling and robust controller-observer for a magnetic microrobot in a fluidic environment using mri gradients," IEEE International Conference on Intelligent Robots and Systems, pp. 534-539, 2009.

[4] G. T. Gillies, R. C. Ritter, W. C. Broaddus, M. S. Grady, M. A. Howard, and R. G. McNeil, "Magnetic manipulation instrumentation for medical physics research," Rev. of Sci. Instruments, vol. 65, no. 3, March 1994.

[5] D. Hays, "Electrostatic adhesion of non-uniformly charged dielectric sphere," Int. Phys. Conf. ser., vol. 118, p. 223228, 1991.

[6] Role of Electrostatics in Adhesion, in Fundamentals of Adhesion, L.-H. Lee, Ed. PLENUM PRESS, 1991.

[7] T. Honda, K. I. Arai, and K. Ishiyama, "Micro swimming mechanisms propelled by external magnetic fields," IEEE Trans. on Magnetics, vol. 32 , no. $5,1996$.

[8] K. Iimura, S. Watanabe, M. Suzuki, M. Hirota, and K. Higashitani, "Simulation of entrainment of agglomerates from plate surfaces by shear flows," Chemical Engineering Science, vol. 64, pp. 1455-1461, 2009.

[9] I. Kanellakopoulos, P. Kokotović, and A. Morse, "A toolkit for nonlinear feedback design," $S C L$, vol. 18, pp. 83-92, 1992.

[10] R. Kehlenbeck and R. D. Felice, "Empirical relationships for the terminal settling velocity of spheres un cylindrical columns," Chemical Eng. Technology, vol. 21, pp. 303-308, 1999.

[11] M. Krstić, I. Kanellakopoulos, and P. Kokotović, Nonlinear and Adaptative Control Design. John Wiley \& Sins, Inc., New York, 1995.

[12] J.-B. Mathieu, G. Beaudoin, and S. Martel, "Method of propulsion of a ferromagnetic core in the cardiovascular system through magnetic gradients generated by an mri system," IEEE Trans. on Biomedical Engineering, vol. 53, no. 2, pp. 292-299, 2006.

[13] J. Mathieu and S. Martel, "In-vivo validation of a propulsion method for untethered medical microrobots using a clinical magnetic resonance imaging system," in IROS, 2007, pp. 502-508.

[14] A.-R. Pries, T.-W. Secomb, and P. Gaehtgens, "Biophysical aspects of blood flow in the microvasculature," Cardiovascular Research, vol. 32(4), pp. 654-667, 1996.

[15] E. G. Quate, K. G. Wika, M. A. Lawson, G. T. Gillies, R. C. Ritter, M. S. Grady, and M. A. H. and, "Goniometric motion controller for the superconducting coil in amagnetic stereoaxis system," IEEE Trans. on Biomed. Eng., vol. 38, no. 9, pp. 899-905, September 1991.

[16] S.-I. Takeda, F. Mishima, S. Fujimoto, Y. Izumi, and S. Nishijima, "Development of magnetically targeted drug delivery system using superconducting magnet," Journal of Magnetism and Magnetic Materials, 2006.

[17] S. Tamaz, R. Gourdeau, A. Chanu, J.-B. Mathieu, and S. Martel, "Real-time mri-based control of a ferromagnetic core for endovascular navigation," IEEE Trans. on Biomed. Eng., vol. 55, no. 7, July 2008.

[18] E. Tijskens, H. Ramon, and J.-D. Baerdemaeker, "Discret element modelling for process simulation in agriculture," Journal of Sound and Vibration, vol. 266, pp. 493-514, 2003.

[19] Y. Tsuji and T. Tanaka, T. Ishida, "Lagragian numerical simulation of plug flow of cohesionless particles in a horizontal pipe," Powder Technology, vol. 71, pp. 239-250, 1992.

[20] P. Vartholomeos and C. Mavroidis, "Simulation platform for selfassembly structures in mri-based nanorobotic drug delivery systems," in IEEE. Int. Conf. on Robotics and Automation, May 2010, to be published.

[21] F. White, Viscous Fluid Flow. McGraw Hill New-York, 1991. 